\title{
Emx2 Is Required for Growth of the Hippocampus But Not for Hippocampal Field Specification
}

\author{
Shubha Tole, ${ }^{1}$ Guy Goudreau, ${ }^{2}$ Stavroula Assimacopoulos, ${ }^{1}$ and Elizabeth A. Grove ${ }^{1}$ \\ 'Department of Neurobiology, Pharmacology, and Physiology, University of Chicago, Chicago, Illinois 60637, and 2Max \\ Planck Institute of Biophysical Chemistry, D-37077 Goettingen, Germany
}

The vertebrate Emx genes are expressed in a nested pattern in early embryonic cerebral cortex, such that a medial strip of cortex expresses Emx2 but not Emx1. This pattern suggests that $E m x$ genes could play a role in specifying different areas or fields of the cortex along the mediolateral axis. Such a role has been supported by the observation that in mice lacking functional Emx2 the hippocampus is shrunken and the most medial field of the cortex, the hippocampal dentate gyrus, appears by cytoarchitecture to be missing (Pellegrini et al., 1996; Yoshida et al., 1997). Use of region-specific molecular markers shows, however, that hippocampal fields are specified and correctly

The hippocampus, like the rest of the cerebral cortex, is divided into cytoarchitectonic areas or fields (Nauta and Feirtag, 1986). Both classic neuronal birth-dating and migration studies, and more recent studies of region-specific gene expression, indicate that the hippocampus is patterned into fields during neurogenesis (Caviness, 1973; Stanfield and Cowan, 1979; Nowakowski and Rakic, 1981; Rakic and Nowakowski, 1981; Tole et al., 1997; Tole and Grove, 1998). However, little is known about the molecular mechanisms that initiate this patterning. It is therefore intriguing that the homeobox genes Emx1 and Emx2 are expressed in a nested pattern in the embryonic cortex, so that a medial strip of cortex is defined by expression of Emx2 but not Emx1 (Simeone et al., 1992a,b). In the hindbrain, nested expression of $H O X$ genes reflects a role in regulating rhombomere identity (Lumsden and Krumlauf, 1996). Nested expression of Emx genes therefore suggests a function in specifying divisions in medial cortex in which the hippocampus develops. Supporting this hypothesis, in mice lacking functional Emx2 the hippocampus is shrunken and the dentate gyrus appears by cytoarchitecture to be missing (Pellegrini et al., 1996; Yoshida et al., 1997). A possible explanation is that nested Emx gene expression is required to specify the neuroepithelium that generates the dentate gyrus. In the absence of Emx2, regional identity is not correctly specified and the dentate gyrus is lost (Pellegrini et al., 1996; Yoshida et al., 1997).

\footnotetext{
Received July 13, 1999; revised Dec. 29, 1999; accepted Jan. 3, 2000.

This work was supported by grants from the Brain Research Foundation, National Institutes of Health (E.G.), the March of Dimes (E.G.), the Max-Planck-Society, and European Union Grant BIO4-CT96-0378 (P. Gruss). We thank Peter Gruss for the gift of Emx2 mutant brains, P. Mason and C. Ragsdale for comments on this manuscript, and Amal Ting for technical assistance. cDNA reagents were provided by E. Boncinelli, G. Lemke, J. Boulter, C. Ragsdale, and S. Nakanishi.

Correspondence should be addressed to E. A. Grove, Department of Neurobiology, Pharmacology, and Physiology, MC0926, University of Chicago, Chicago, IL 60637. E-mail: egrove@drugs.bsd.uchicago.edu.

Dr. Tole's present address: Tata Institute of Fundamental Research, Homi Bhabha Road, Colaba, Mumbai 400,005, India.

Copyright (C) 2000 Society for Neuroscience $\quad 0270-6474 / 00 / 202618-08 \$ 15.00 / 0$
}

positioned in the Emx2 mutant. In particular, a dentate cell population is generated, although it fails to form a morphological gyrus. This failure may be part of a more widespread medial cortical defect in the mutant. Examination of cortical cell proliferation and differentiation indicates a disruption of the maturation of the medial cortex in the absence of Emx2. Thus, Emx2 is required for normal growth and maturation of the hippocampus but not for the specification of cells to particular hippocampal field identities.

Key words: Emx2; hippocampus; patterning; specification; cortical maturation; cortical hem

Reports that specific mutations lead to morphological defects in the hippocampus (Xuan et al., 1995; Pellegrini et al., 1996; Porter et al., 1997; Yoshida et al., 1997) raise a need for molecular markers of different hippocampal fields. Analysis by cytoarchitecture alone may be misleading in that the cells that make up a particular field may be present in a mutant but reduced in number or abnormally organized so that the field cannot be detected histologically. This distinction is significant because the complete and selective loss of a field suggests that the mutated gene is needed to specify cells to a particular field identity. In contrast, reduction of the size of a field, particularly in the context of general cortical shrinkage or dysmorphology, suggests a different role for the gene in question, perhaps in hippocampal growth or assembly.

Using a panel of region- and field-specific hippocampal molecular markers, we reanalyzed the Emx2 mutant mouse line described by Pellegrini et al. (1996). Because the hippocampus in the Emx2 mutant mouse is smaller than in wild-type mice (Pellegrini et al., 1996; Yoshida et al., 1997), we asked not only whether dentate gyrus cells are missing, but whether additional fields are lost. We report that cells are specified to form all the major hippocampal fields, including a dentate field. Emx2 is thus not required to specify cells to a dentate field identity but is required for the formation of a morphological dentate gyrus. Further observations suggest a general requirement for Emx2 for the timely growth and maturation of the hippocampus and adjacent medial neocortex.

\section{MATERIALS AND METHODS}

Emx2 mutant mice were generated and genotyped as described previously (Pellegrini et al., 1996). In brief, the Emx2 gene was mutated by homologous recombination to produce mutant $\mathrm{C} 57 \mathrm{BL} / \mathrm{J} 6$ mice carrying a null allele. The targeting construct used was a classical replacement vector designed so that the neomycin resistance gene replaced the second and part of the third helix of the Emx2 homeobox domain (for details, see Pellegrini et al., 1996). Homozygous Emx2 mutant embryos (Emx2 -imice) and their littermates were recovered for gene expression analysis at 


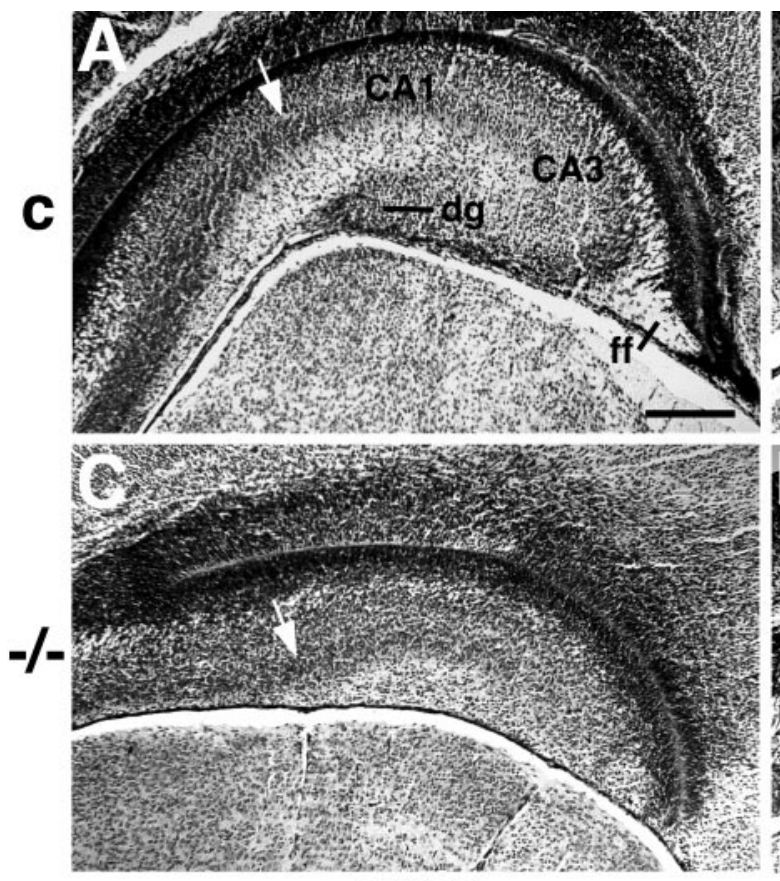

E16.5
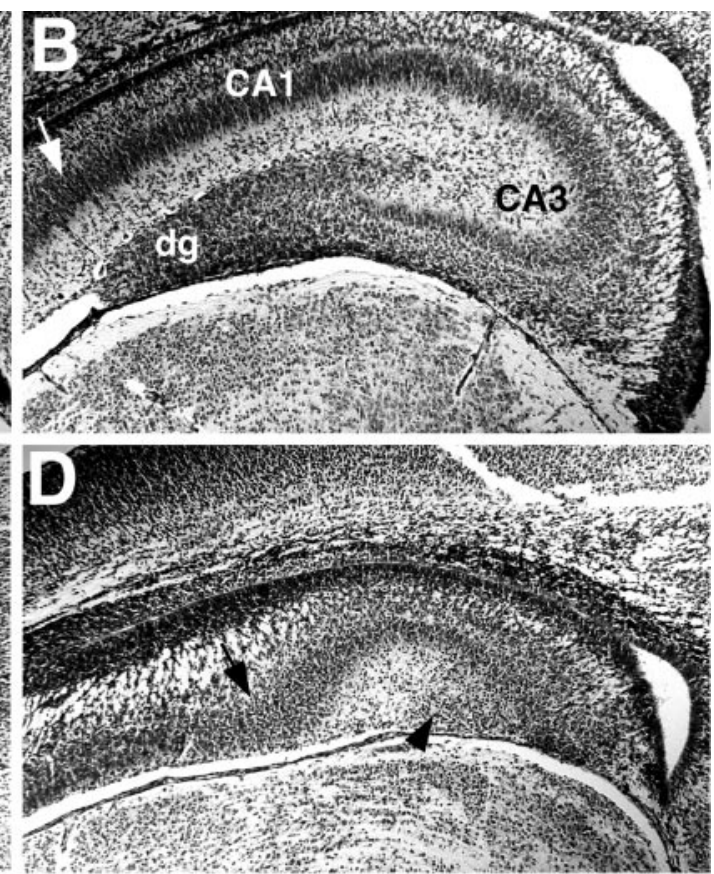

E18.5

Figure 1. The hippocampus is reduced in size in the Emx2 mutant and has no morphologically identifiable dentate gyrus. $A-D$, Nissl-stained coronal sections through the hippocampus of wild-type $(A, B)$ or Emx2 homozygous $(-/-)$ mutant mice $(C, D)$. Arrows indicate the approximate boundary between the embryonic hippocampal pyramidal cell layer and the adjacent subiculum. $A$, $C$, Sections from E16.5 littermate mice, matched for rostrocaudal level. The hippocampal pyramidal cell layer is shorter in the Emx2 mutant $(C)$ than in the wild-type embryo $(A)$. The dentate gyrus $(d g)$ and fimbria fornix ( $f f$ ) have begun to develop in the wild-type embryo $(A)$, but no dentate gyrus can be identified in the mutant and the fimbria fornix is severely shrunken $(C) . B, D$, Sections from E18.5 mice, matched for rostrocaudal level. The size of the hippocampus is markedly different between a wild-type embryo $(B)$ and an $\operatorname{Emx} 2$ mutant $(D)$. At E18.5, a morphological dentate gyrus is clearly identifiable in the wild-type embryo $(B)$ but not detectable in the mutant $(D)$. Arrowhead in $D$ indicates a small patch of cells that may represent a residual dentate cell population (compare cells indicated by arrows in Fig. $2 K, L)$. Scale bar: $A-D, 150 \mu \mathrm{m}$.

embryonic day 14.5 (E14.5), E16.5, or E18.5. Additional wild-type mice were harvested at E10.5 and E12.5 to analyze expression of Emx1 and Emx2. Midday of the day of vaginal plug discovery was considered E0.5. Birth, for the mouse strains examined, occurs at approximately E19.5.

Brains were fixed and processed for two-color whole-mount in situ hybridization, or sectioned and processed for single-color in situ hybridization as described previously (Tole and Patterson, 1995; Grove et al., 1998). Probes used for in situ hybridization were specific for Emx1, Emx2, class III $\beta$-tubulin, TTR, Wnt2b, Wnt3a, SCIP, KA1, NK3, Ephb1, Steel, and iGluR7 (Shigemoto et al., 1990; Simeone et al., 1992b; Tole et al., 1997; Grove et al., 1998; Tole and Grove, 1998; Lee et al., 2000). Dividing cells in mouse embryos were labeled with 5-bromo- $2^{\prime}$-deoxyuridine (BrdU) $(100 \mathrm{mg} / \mathrm{kg}$; Roche Diagnostics Corp., Indianapolis, IN) delivered intraperitoneally to pregnant mice $2-4 \mathrm{hr}$ before they were killed. Nucleotide incorporation on tissue sections was detected with antibody M0744 (Dako, Carpinteria, CA), followed by diaminobenzidine peroxidase immunohistochemistry. For Nissl staining, embryo heads or brains were dehydrated and embedded in paraffin, and $10 \mu \mathrm{m}$ coronal sections were obtained with a rotary microtome. Sections were stained with cresyl violet.

A total of $29 \mathrm{Emx} 2^{-1-}$ mouse brains were analyzed at E14.5 $(n=4)$, E16.5 $(n=7)$, or E18.5 $(n=18)$ and compared with equal numbers of wild-type or heterozygote mutant littermate mice. E18.5 is $1 \mathrm{~d}$ before birth and was selected as the primary age of analysis for several reasons. First, Emx2 $2^{-1-}$ mice lack kidneys and die within a few hours of birth (Pellegrini et al., 1996; Yoshida et al., 1997). Thus, selecting E18.5 for analysis allows the hippocampus to develop as long as possible and gives the best opportunity of identifying different hippocampal fields in the mutant. The major hippocampal fields are readily identifiable by either cytoarchitecture or molecular markers at E18.5. A morphological dentate gyrus is evident in wild-type mice by E16.5, and a range of molecular markers distinguish different hippocampal fields and subregions by E15.5 (Caviness, 1973; Stanfield and Cowan, 1979; Tole et al., 1997; Tole and Grove, 1998). Finally, previous analyses of hippocampal morphology in
Emx2 -/- mice were made at E18.5-E19.5 (Pellegrini et al., 1996; Yoshida et al., 1997). Thus, our observations could be compared directly with previous findings. Additional litters were harvested at younger ages to compare the overall development of the cerebral cortex in mutant and control mice. No differences were observed in cortical morphology or gene expression patterns between wild-type and heterozygote Emx2 mutant mice, which were therefore pooled as controls.

\section{RESULTS}

During the early period of corticogenesis, Emx2 is expressed in the ventricular zone (VZ) of the embryonic cerebral hemisphere (Simeone et al., 1992a,b; Gulisano et al., 1996). The gross morphology of the Emx $2^{-1-}$ brain is consistent with this pattern of Emx2 expression. The cerebral hemispheres of Emx $2^{-1-}$ mice are reduced in size compared with wild-type mice (Pellegrini et al., 1996; Yoshida et al., 1997), and these gross changes are apparent by E11.5 (Yoshida et al., 1997).

In Nissl-stained sections, the medial part of the Emx2 -/mutant cerebral cortex shows particularly marked defects (Pellegrini et al., 1996; Yoshida et al., 1997; Fig. 1). At E16.5, the hippocampal pyramidal layer appears shorter in the mutant than in wild-type mice (Fig. 1, compare $A, C$, sections matched for rostrocaudal level). In wild-type mice at this age, both the embryonic dentate gyrus and the fimbria fornix are beginning to develop (Fig. $1 A$ ), but in the Emx $2^{-/-}$mutant, no dentate gyrus can be seen and the fimbria fornix is barely detectable (Fig. 1C). At E18.5, the size difference between the mutant and wild-type hippocampus is more striking (Fig. 1, compare $B, D$, sections matched for rostrocaudal level). A morphological dentate gyrus is 


\begin{tabular}{lllll}
\hline Table 1. & Molecular markers of subregions in the mouse hippocampus at E18.5 & \\
& $\begin{array}{l}\text { Function of encoded } \\
\text { protein }\end{array}$ & $\begin{array}{l}\text { Fields } \\
\text { marked }\end{array}$ & $\begin{array}{l}\text { Same fields } \\
\text { marked in adult? }\end{array}$ & $\begin{array}{l}\text { Fields marked } \\
\text { in Emx2 mutant }\end{array}$ \\
\hline $\begin{array}{l}\text { Steel } \\
\text { SCIP }\end{array}$ & $\begin{array}{l}\text { c-kit ligand } \\
\text { POU-domain transcription } \\
\text { factor }\end{array}$ & $\begin{array}{l}\text { DG, CA1 } \\
\text { CA1 }\end{array}$ & $\begin{array}{l}\text { DG, yes } \\
\text { yes }\end{array}$ & DG, CA1 \\
KA1 & Glutamate receptor subunit & CA3 & yes & CA3 \\
$i G l u R 7$ & Glutamate receptor subunit & DG, CA1 & no & DG, CA1 \\
$W n t 5 a$ & Wnt protein & DG & no & DG \\
$E p h b 1$ & Ephrin receptor & DG/CA3* & no & DG/CA3 \\
$N K 3$ & Neuromedin K receptor & CA1/CA3* & no & CA1/CA3
\end{tabular}

*Slash indicates that a marker labels a continuous region that incorporates parts of two fields. DG, Dentate gyrus.
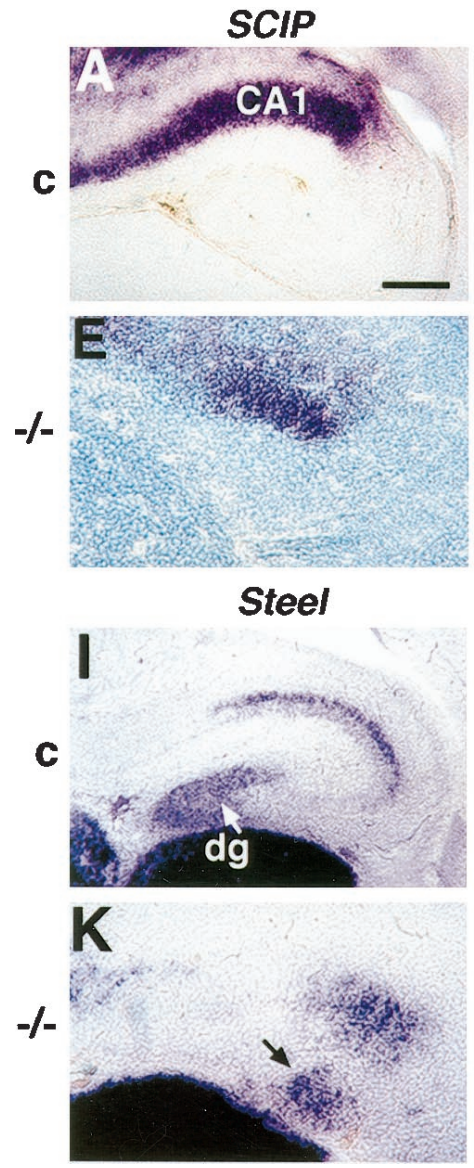
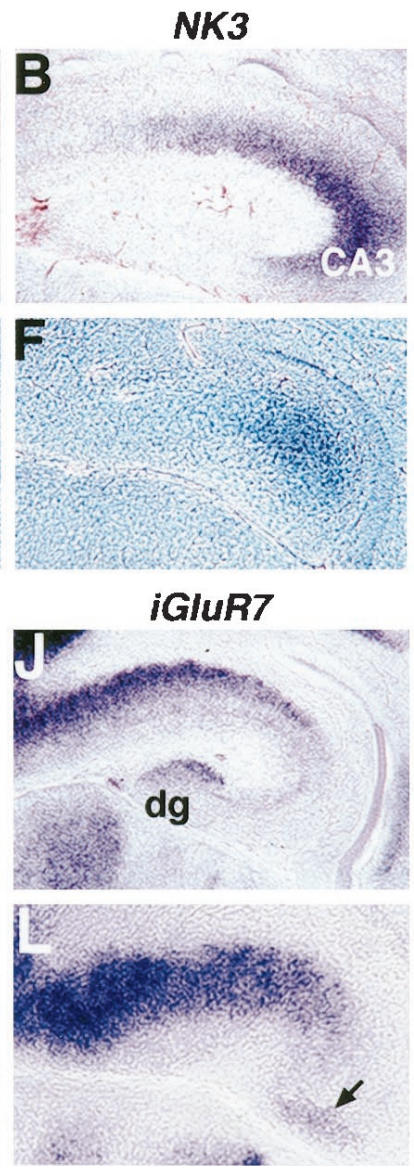
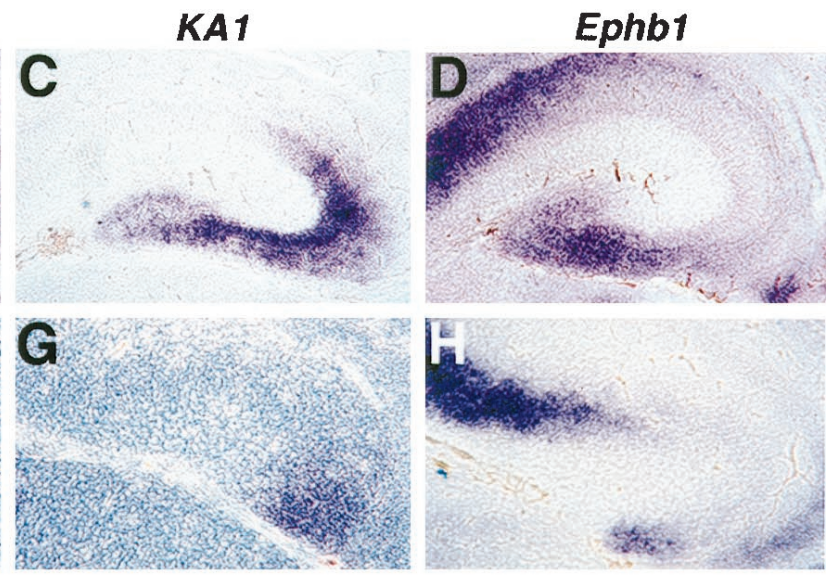

Figure 2. Field specification is preserved in the hippocampus of Emx 2 mutant mice, and a dentate cell population is present. $A-L$, Coronal sections through the hippocampus of E18.5 control $(c)$ $(A-D, I, J)$ or Emx2 homozygous (-/-) mutant mice $(E-H, K, L)$ processed for in situ hybridization. Medial is to the left. $A-D$, In control brains, expression of SCIP appears in CA1 and adjacent extrahippocampal cortex $(A), N K 3$ in contiguous parts of CA1 and CA3 $(B), K A 1$ in CA3 $(C)$, and Ephb1 in the dentate gyrus and a part of CA3 close to the dentate (D). E-H, In Emx2 mutant brains, $S C I P, N K 3, K A 1$, and Ephb1 are expressed in the same relative positions as in control brains, but each marked territory or field is smaller in the mutant. I-L, In the hippocampus of control mice, the developing dentate gyrus $(d g)$ is marked by expression of Steel $(I)$ and $i G l u R 7(J)$. Steel expression, in particular, reveals the developing V-shape of the dentate gyrus. In the Emx2 mutant hippocampus, Steel $(K)$ and $i G l u R 7(L)$ mark patches of dentate cells (arrows) that do not form a gyrus. Scale bar: $A-D, 150 \mu \mathrm{m} ; E-H, K, L, 120 \mu \mathrm{m} ; I, J, 200 \mu \mathrm{m}$. clear in the wild-type hippocampus (Fig. $1 B$ ) but still cannot be distinguished in the mutant (Fig. 1D).

To analyze further the development of fields and subregions in the hippocampus in the Emx $2^{-/-}$mutant, we assembled a panel of molecular markers. These markers allow the hippocampal CA fields and the dentate gyrus to be identified and distinguished from one another in the embryonic mouse hippocampus beginning between E14.5 and E15.5 (Table 1) (Tole et al., 1997). Some markers are permanently expressed in particular fields. For example, beginning at E14.5-E15.5 and continuing into adulthood, $K A 1$ and $S C I P$ are expressed in the pyramidal neurons of CA3 and CA1, respectively, and expression of Steel marks the granule cell layer of the dentate gyrus (Fig. 2A, C, I; Table 1) (Motro et al., 1991; Wisden and Seeburg, 1993; Frantz et al., 1994; Tole et al.,
1997; Lee et al., 2000). Other markers are transient, distinguishing hippocampal subregions only in the embryo and neonate (Table 1). For example, at E18.5, the embryonic age selected for primary analysis of the Emx 2 mutant, $N K 3$ is expressed in a region that consists of parts of CA1 and CA3, Wnt5a and iGluR7 are expressed in the developing dentate gyrus, and the ephrin receptor Ephb1 is expressed in the dentate gyrus and a contiguous part of CA3 (Fig. 2B, D, J; Table 1).

Region- and field-specific hippocampal markers were detected by in situ hybridization at E18.5 in each Emx $2^{-1-}$ mutant brain examined $(n=16)$, and expression of each marker appeared in the correct relative position, despite marking a much smaller territory than in control brains (Table 1; Fig. $2 E-H$ ). In particular, markers of the dentate pole of the hippocampus appeared. 
These included expression of Ephbl (Fig. 2H), as well as specific markers of the dentate gyrus: Steel, iGluR7, and Wnt5a (Table 1; Fig. $2 K, L)$. Thus, interdigitating expression of SCIP, NK3, KA1, Ephb1, Steel, iGluR7, and Wnt5a indicates that CA1, CA3, and dentate fields are developing in the Emx2 mutant but that each field is smaller than in control brains. Specification of cells for each hippocampal field, including the generation of a dentate gyrus cell population, therefore appears to be preserved in the mutant.

Despite the presence of a dentate gyrus cell population, the gyrus itself, a gross morphological structure, cannot be identified in the Emx2 mutant (Pellegrini et al., 1996; Yoshida et al., 1997) (Fig. 1) The dentate cells that are labeled with molecular markers in the E18.5 mutant appear in amorphous patches rather than forming a distinct gyrus as in control mice (Fig. 2). Could this defect reflect a widespread dysmorphology in the Emx2 mutant cortex, or one that is specific to the dentate? To address this question, several features of general cortical development were compared between Emx2 mutants and wild-type mice at a range of ages.

Analysis of SCIP expression suggests a shared defect in the hippocampus and neocortex of the Emx $2^{-1-}$ mutant. In wildtype mice, SCIP expression labels a subpopulation of postmitotic neurons in both hippocampus and neocortex (He et al., 1989; Frantz et al., 1994; Tole et al., 1997). Initially, SCIP-expressing cells are arrayed in a diff use, broad band as they leave the VZ and migrate toward the cortical plate (Frantz et al., 1994; Tole et al., 1997). At E18.5, however, most SCIP-expressing cells have settled in the cortical plate, indicated by a dense band of SCIP labeling in both neocortex and hippocampus (Fig. $3 C$ ). In contrast, in the Emx2 mutant cortex at E18.5, SCIP labeling is diff use (Fig. $3 F$ ), resembling the pattern seen 2-3 d earlier in control brains (Fig. 3, compare $F, I)$. This abnormal pattern of SCIP labeling could result from a misregulation of $S C I P$ in the Emx2 $-1-$ mutant. However, an alternative explanation is that cells express SCIP correctly in the Emx $2^{-/-}$mutant but that neuronal migration is disrupted or delayed, giving the pattern of SCIP expression an immature appearance.

Because cortical neurons take 2-3 d to migrate from the VZ to the cortical plate (Altman and Bayer, 1990c), defects in the cortex at E18.5 could be attributable to abnormalities in corticogenesis occurring at least $2 \mathrm{~d}$ earlier. To identify cells that would generate the postmitotic cells labeled with our panel of markers at E18.5, we injected BrdU into pregnant mice at E14 or E16.5 and harvested the labeled embryos 2-4 hr later. This labeling protocol provides a snapshot of cells that were dividing just before tissue harvest.

The pattern of cell proliferation in the Emx2 mutant mouse, like the pattern of SCIP expression, appears disrupted and perhaps delayed. At E16.5, cortical neurogenesis is almost over in the mouse (Angevine, 1965). In both the hippocampus and neocortex of control mice, a compact band of BrdU-labeled cells is all that remains of the $\mathrm{VZ}$ (Fig. $3 A, B$ ). In contrast, in $E m \times 2^{-/-}$mutants at E16.5, the BrdU-labeled VZ represents a large proportion of the total thickness of the embryonic cortex (Fig. 3D,E). Such a pattern of BrdU labeling in which the zone of dividing VZ cells is broad relative to the total thickness of the embryonic cortex is reminiscent of the pattern seen 2-3 d earlier in control mice (Fig. 3, compare $D, E$ with $G, H)$. The abnormally broad VZ at E16.5 could be generated in several ways, including a delay in cortical neurogenesis or a failure of cells to leave the cell cycle on schedule and migrate away from the VZ. Whatever the cause, the cortex again displays an immature appearance in the Emx2 mutant compared with littermate controls.

Neurons continue to be generated for the dentate gyrus into postnatal life, and late-born dentate cells are generated from secondary germinal zones rather than from the VZ (Angevine, 1965; Caviness, 1973; Stanfield and Cowan, 1979; Altman and Bayer, 1990a,b). In E16.5 control brains labeled with BrdU, many BrdU-positive cells appear along the subpial face of the hippocampus (Fig. $3 A$ ) in which a secondary dentate germinal population has been identified (Angevine, 1965; Caviness, 1973; Stanfield and Cowan, 1979; Altman and Bayer, 1990a,b). Only sparse BrdU-labeled cells are detectable in a corresponding location in the Emx2 mutant (Fig. 3D), suggesting that this latedividing progenitor population is still small. The dentate cells identifiable at E18.5 in the mutant may therefore have been generated almost exclusively by primary progenitors in the VZ, partially explaining the small size of the dentate cell population.

These observations indicate that the dentate field of the hippocampus is specified in the Emx2 mutant mouse but suggest that its development may be retarded. Consistent with this interpretation, the appearance of another hippocampal field is also retarded in the mutant. At E14.5, embryonic CA3 is identifiable by $K A 1$ expression in control brains (Fig. $3 J$ ). In contrast, CA3 cannot be detected by $K A 1$ expression at E14.5 in the Emx2 mutant brain (Fig. $3 M$ ), although this field is readily detected in the mutant at E18.5 (Fig. 2G).

Slowed appearance of a CA3 field marker may reflect a general slowing of cortical neuronal differentiation in the Emx2 -/mutant. The peak of such differentiation in the mouse occurs between E11 and E18 (Caviness, 1973; Caviness and Sidman, 1973) when postmitotic neurons migrate from the VZ to form an increasingly thick preplate-cortical plate. In control brains at E14.5, the forming preplate-cortical plate is indicated by dense expression of class III $\beta$-tubulin, a general neuronal marker (Fig. $3 K, L)$. Beneath this dense band of labeling, numerous more dispersed neurons, presumably in the process of migrating away from the VZ, also express class III $\beta$-tubulin (Fig. $3 L$ ). In Emx2 ${ }^{-1-}$ mutant cortex at the same age, the class III $\beta$-tubulin-dense band is thinner (Fig. $3 N, O$ ), and there are fewer dispersed $\beta$-tubulin-expressing cells (Fig. 3O, arrows). A diminished preplate-cortical plate characterizes a large medial region of the mutant cortex that includes both hippocampus and dorsal neocortex (Fig. 3, compare $K, N$ ).

These and previous observations (Pellegrini et al., 1996; Yoshida et al., 1997) suggest that Emx2 is directly required for the normal growth and maturation of the medial cortex. A formal possibility, however, is that the small size and immature appearance of the cerebral hemispheres and hippocampus in particular is part of a systemic effect of the Emx2 mutation that leads to general shrinkage and developmental delay. Arguing against this possibility, other brain regions, such as the brainstem, are not grossly smaller in size in Emx $2^{-1-}$ mutants than in wild-type mice (Pellegrini et al., 1996; Yoshida et al., 1997). To test this possibility further, we measured the body size of Emx2 -/embryos and littermate controls and compared their general morphological development using the staging system of Theiler (1989). No size differences were found between Emx $2^{-/-}$mutant bodies and heterozygotes or wild-type embryos; furthermore, mutant and control embryos were indistinguishable with respect to stage-specific external features described by Theiler (1989), such as digit and skin development.

Findings of the present study suggest that the Emx2 mutation 
Figure 3. Disruptions of medial cortical development in the Emx2 mutant. $A-O$, Coronal sections through the hippocampus of control $(A-C, G-I, J-L)$ or Emx2 mutant mice $(D-F, M-O) . B, E$, $H, L$, and $O$ show portions of $A, D, G, K$, and $N$, respectively, at higher magnification. $A, B, D, E, G, H$, Dividing cells were labeled with BrdU $4 \mathrm{hr}$ before brains were fixed. BrdU-labeled cells appear dark brown; light brown staining is non-specific. In a control mouse at E16.5, BrdU-labeled cells mark a narrow ventricular zone $(v z)$ in the hippocampus and neocortex $(A, B)$. Above the vz in neocortex, scattered BrdUlabeled cells appear in the developing subventricular zone $(s v z)$, a second germinal layer that generates glia $(A, B)$. The subventricular zone is not prominent in the hippocampus $(A, B)$. In an Emx2 mutant at E16.5, in contrast, BrdU-labeled cells mark a broad vz in the hippocampus and neocortex, and a separate subventricular zone is hard to distinguish $(D, E)$. A similarly broad $\mathrm{vz}$ relative to the total thickness of the embryonic cortex is seen at E14 in a control mouse $(G, H)$. Arrows in $B, E$, and $H$ indicate the rough boundaries of the $\mathrm{vz}$, on either side of the ventricle, in the hippocampus and an overlying part of neocortex. Arrowhead in $A$ indicates a population of BrdU-labeled cells likely to represent a secondary dentate precursor pool that develops late in hippocampal neurogenesis. Only sparse BrdUlabeled cells appear in a corresponding position in the Emx 2 mutant hippocampus (arrowhead in $D) . C, F, I$, In a control cortex at E18.5, SCIP-expressing cells form a compact cortical plate $(C)$. $S C I P$-expressing cells in an Emx $2 \mathrm{mu}-$ tant cortex are more dispersed $(F)$, as are SCIP-expressing cells in a control cortex $3 \mathrm{~d}$ earlier, at E15.5 (I). $J, M$, At E14.5, in a control brain, embryonic CA3 is marked by expression of KA1 (arrow in $J$ ). KA1 is not yet expressed in the hippocampus of an Emx2 mutant at this age (arrow in $M$ ) but is expressed in the thalamus (th). $K, L, N, O$, At E14.5, in a control cortex, a broad, dense band of cells expresses class III $\beta$-tubulin mRNA (dark blue), a marker of neuronal differentiation $(K)$. Beneath this dense band, numerous, more scattered cells also express class III $\beta$-tubulin (arrows indicate some of these cells in $L$ ). The dense band of differentiating neurons is less developed in a large medial region of the cortex in an Emx2 mutant (compare cortex medial to marked lines in $K$ and $N$ ). Only sparse cells beneath this dense band express class III $\beta$-tubulin (arrows in $O$ ). Scale bar: $A, C, D, F, G, I, 230 \mu \mathrm{m} ; B, E, H, 175$ $\mu \mathrm{m} ; J, K, M, N, 200 \mu \mathrm{m} ; L, O, 70 \mu \mathrm{m}$.

disrupts medial cortical development, but that the dentate gyrus is not selectively affected. Perhaps, however, early nested expression of Emx genes defines a cortical region other than the developing dentate gyrus, and it is this structure that is selectively affected when nested Emx1/2 expression is lost. To address this possibility, we characterized the region of medial cortex that expresses $E m x 2$ but not Emx1 at E10.5-E12.5, early in the period of cortical neurogenesis. At E12.5, the medial edge of the cerebral hemisphere has begun to differentiate into choroid plexus epithelium $(\mathrm{CPe})$, which is marked by strong transthyretin (TTR)
BrdU
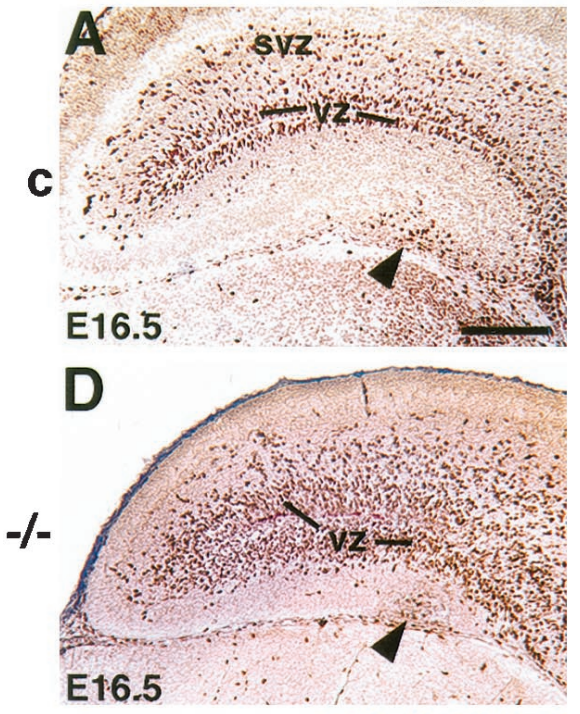

C

\section{E14}

E14.5
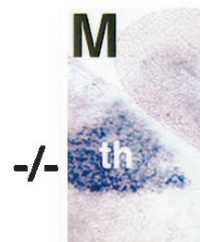

E14.5
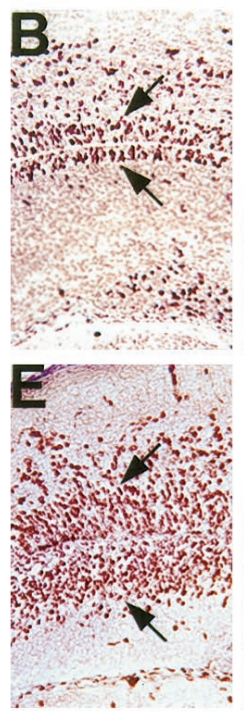

E18.5
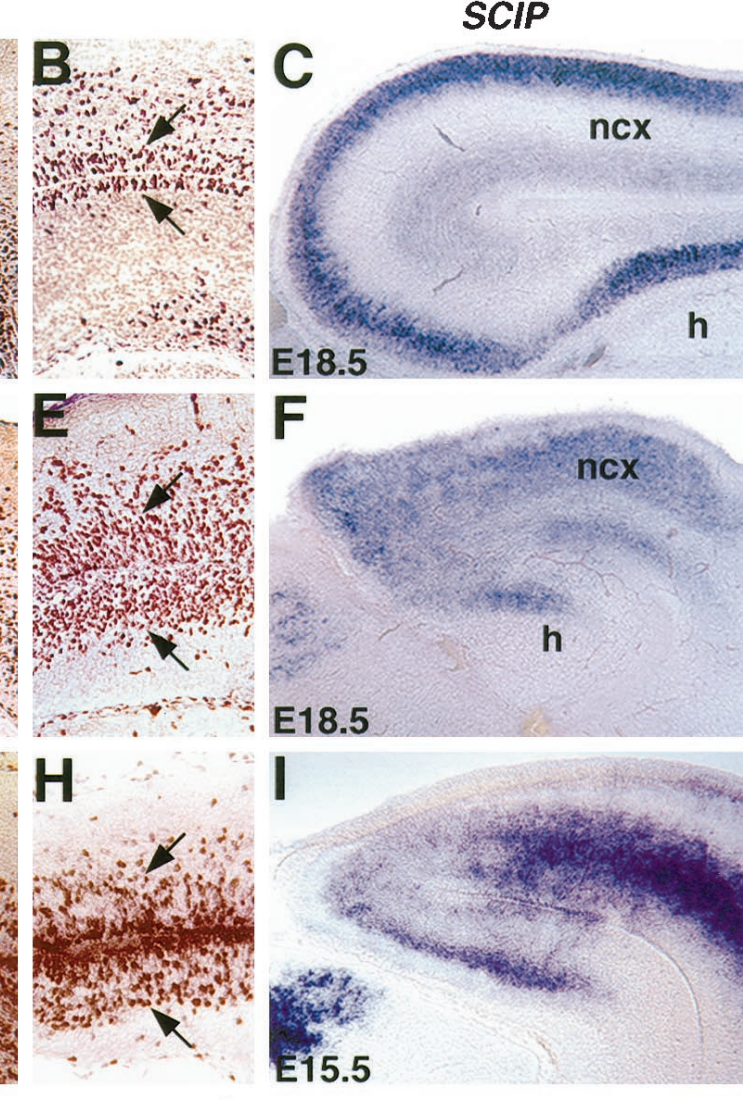

$\beta-t u b$
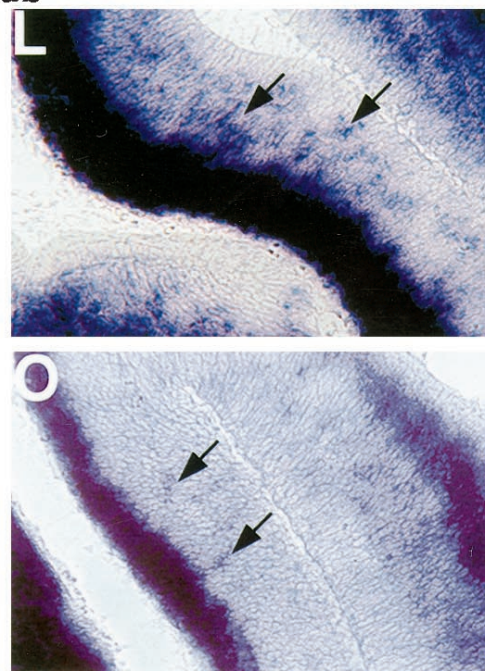

expression from the earliest stages of its development (Thomas and Dziadek, 1993). The CPe therefore serves as an easily identifiable medial landmark with which the borders of Emxl and Emx2 expression can be compared. Two-color Emx1-TTR in situ hybridization at E12.5 reveals a band of Emx1-poor neuroepithelium dorsal and caudal to the CPe (Fig. 4A). Emx2 is expressed in this Emx1-poor band, but not in the CPe or in junctional epithelium at the border of the CPe (Fig. 4D). The outline of the Emxl-poor band (Fig. 4A) strongly resembles that of the cortical hem, an embryonic structure that expresses multiple $W n t$ genes 

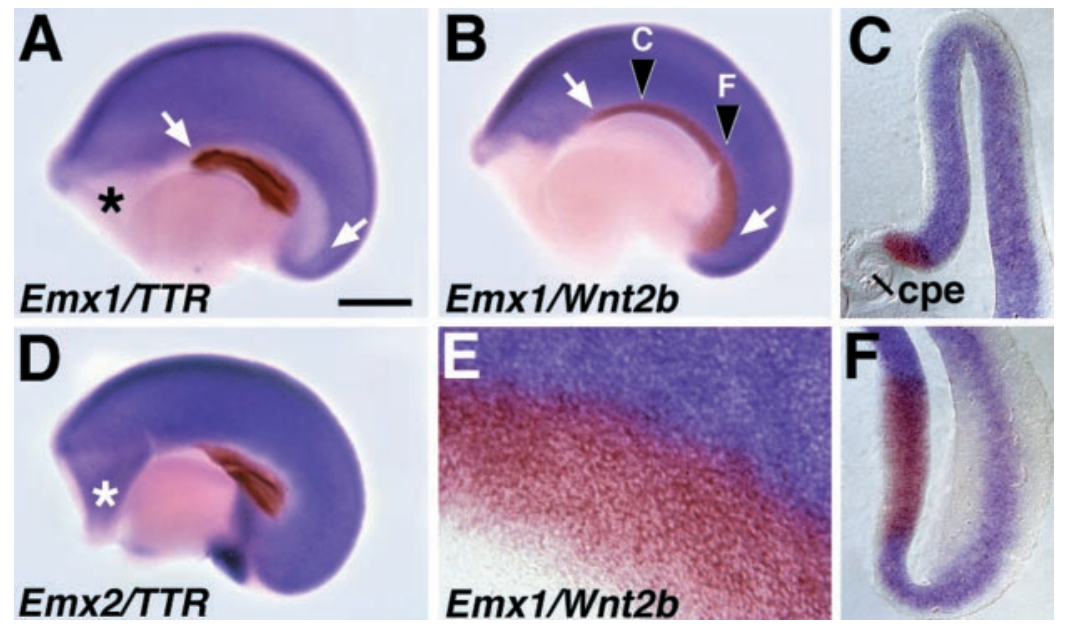

Figure 4. Nested expression of Emx1 and Emx2 at E12.5 defines the cortical hem. $A, B, D, \mathrm{E} 12.5$ cerebral hemispheres from wild-type mice, processed as whole mounts for twocolor in situ hybridization, viewed from the medial side. Rostral is to the left. E, A higher magnification view of one such hemisphere. $C, F$, Coronal sections through a hemisphere at the rostrocaudal levels marked in $B . A, D$, The choroid plexus epithelium (cpe) is marked by expression of TTR (brown). Emx1 expression (blue in $A$ ) avoids a band of cortical neuroepithelium curving dorsal and caudal to the cpe (arrows) and a rostral telencephalic region (asterisk). Emx2 (blue in $D$ ) is expressed in both of these Emx1-poor regions. $E m x 2$ is not expressed in the cpe or in a narrow strip of junctional epithelium (white in $D$ ) adjacent to the cpe $(D) . B$, $C, E, F, W n t 2 b$ expression (brown) fills the curving band of cortical neuroepithelium that is Emxl-poor $(B, C, E, F)$ and defines the cortical hem. At the boundary of the cortical hem, $W n t 2 b$ and Emxl expression may overlap by one or two cell widths, best seen at higher magnification $(E)$. The rostral telencephalic region that is also Emxl-negative (asterisks in $A$ and $D$ ) is not part of the cortical hem. Scale bar: $A, B, D, 500$ $\mu \mathrm{m} ; E, 65 \mu \mathrm{m} ; C, F, 170 \mu \mathrm{m}$.
(Grove et al., 1998). Two-color Emx1-Wnt2b in situ hybridization confirms that this band is filled by $W n t 2 b$ expression and is therefore the Wnt-expressing cortical hem (Fig. 4B,C,E,F). At E10.5, expression of Emx1 and Emx2 in the dorsal telencephalon appears similarly nested, with Emx2 expression more extensive than Emx1 (Simeone et al., 1992b). Expression of Wnt3a, which marks the incipient cortical hem at this age, fills in the Emx2positive/Emxl-negative zone (data not shown).

At early stages of cortical neurogenesis, therefore, the region of dorsomedial cortical neuroepithelium that expresses Emx2 but not Emx1 is the cortical hem (Fig. 5). The cortical hem has not been fate mapped; thus, the cell types it generates have not been definitively identified. However, the cortical hem does not include regions of neuroepithelium described previously as generating hippocampal neurons (Angevine, 1965; Caviness, 1973; Stanfield and Cowan, 1979; Altman and Bayer, 1990a,b,c). Most relevant, the identified primary and secondary dentate neuroepithelia (Angevine, 1965; Caviness, 1973; Stanfield and Cowan, 1979; Altman and Bayer, 1990a,b) lie outside the cortical hem (Grove et al., 1998). Thus, the lack of a selective effect on a particular subfield of the hippocampus in the Emx2 mutant may be consistent with the normal early patterns of expression of Emx1 and Emx2.

The cortical hem may, however, regulate the development of adjacent structures by secreting Wnt and Bmp proteins (Furuta et al., 1997; Grove et al., 1998). In particular, even if the cortical hem does not contribute neurons directly to the hippocampus, a Wnt3a signal from the hem is required for normal hippocampal development (Lee et al., 2000). If nested expression of Emx genes plays a role in cortical regional specification, the cortical hem might be selectively lost in the Emx $2^{-/-}$mutant mouse, and this, in turn, could lead to a smaller hippocampus. Expression of $W n t 2 b, W n t 3 a$, and $W n t 5 a$ shows, however, that the cortical hem is present in the mutant (Yoshida et al., 1997; data not shown). The hem region appears expanded early in development (Yoshida et al., 1997) but slightly shrunken at later embryonic ages (data not shown), consistent with the overall reduction of the Emx2 -/cortical mantle. Previous studies suggest that the cortical hem generates CPe cells and glial cells of the fimbria fornix (Maruyama and D'Agostino, 1967; Sturrock, 1979; Zaki, 1981; Altman and Bayer, 1990b; MacKenzie et al., 1991; Nicholson-Flynn et al., 1996). Consistent with such an origin for the CPe, the Emx2 mutant has an abnormally small CPe (Pellegrini et al., 1996;
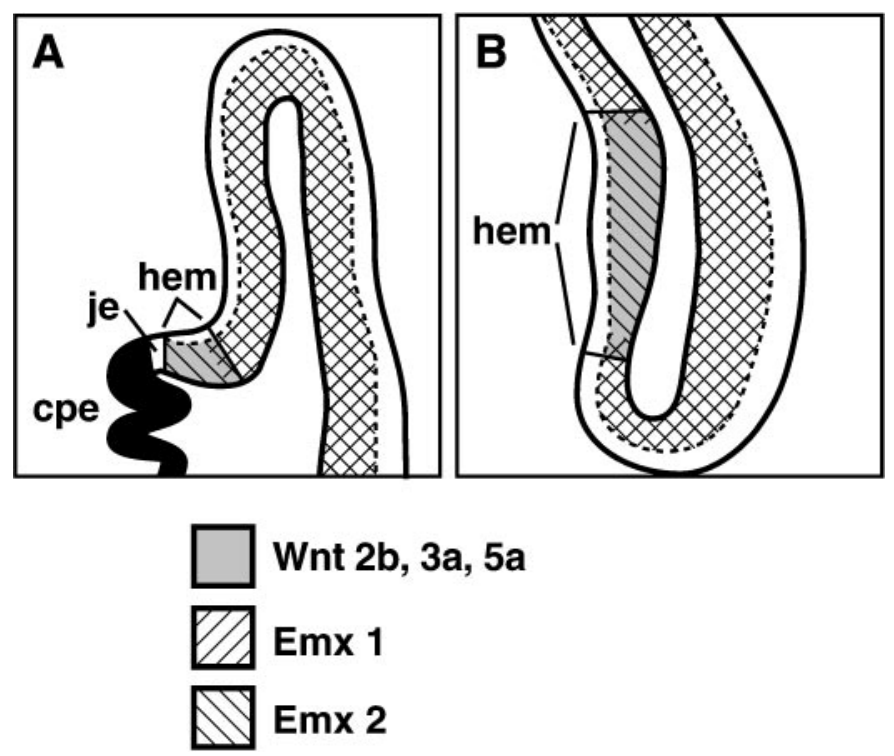

Figure 5. Summary of gene expression patterns with respect to the cortical hem. Schematic coronal sections through an E12.5 cerebral hemisphere at midrostral $(A)$ and caudal $(B)$ levels. At E12.5, the ventricular zone of the embryonic cerebral cortex expresses Emx2. The territories of Emx1, Wnt2b, Wnt $3 a$, and Wnt5a expression are nested within the region of Emx2 expression. Wnt2b, Wnt3a, and Wnt5a are expressed in the cortical hem only; expression of Emx1 fills most of the cortical neuroepithelium, but not the cortical hem. Expression of at least two other transcription factors, $B F-1$ and $L h x 2$, resembles that of Emx 1 in avoiding the cortical hem. Selectively low expression of these genes may in part reflect the fate of the cortical hem to shrink with respect to the rest of the cortical neuroepithelium (see Discussion). cpe, Choroid plexus epithelium; hem, cortical hem; je, junctional epithelium adjacent to the differentiating cpe.

Yoshida et al., 1997; data not shown). The fimbria fornix is also shrunken in the Emx2 mutant (Pellegrini et al., 1996; Yoshida et al., 1997), although this is an expected secondary effect of a reduced hippocampus. Thus, the cortical hem and its likely derivatives are present in the Emx2 mutant.

\section{DISCUSSION}

Cytoarchitectural analysis of the Emx2 mutant shows a dramatic loss of the hippocampal dentate gyrus and a shrinkage of the 
entire hippocampus (Pellegrini et al., 1996; Yoshida et al., 1997). Because nested Emx gene expression defines the most medial portion of the embryonic cerebral cortex, a plausible hypothesis is that nested Emx1/2 expression is important for specifying the region that gives rise to part of the hippocampus, prominently including the dentate gyrus. Thus, in the Emx2 mutant, dentate cells, and perhaps cells for other hippocampal fields, are never specified. Our observations indicate, however, that although each field is smaller in the Emx2 mutant, populations of cells are specified for each field, including the dentate. These populations develop in the correct positions relative to one another. Additional observations identify the region of dorsomedial cortical neuroepithelium that is defined by nested $E m \times 1 / 2$ expression as the Wnt-rich cortical hem. Wnt gene expression indicates that this structure too is present in the Emx2 mutant. Thus, neither the nested expression of Emx1/2 nor Emx2 itself are absolutely required to specify cells to different regional identities at the medial edge of the cerebral cortex.

Although each of the major medial regions is present in the Emx2 mutant, each region appears abnormally small (Pellegrini et al., 1996; Yoshida et al., 1997; present study). For the hippocampus, this defect could result in part from reduction of growth signals from a slightly shrunken cortical hem. However, the sector of medial cortex that is reduced in the Emx2 mutant is larger than the hippocampus alone (Pellegrini et al., 1996; Yoshida et al., 1997; present study), suggesting that the hippocampal defect is part of a more widespread effect of the Emx2 mutation. The disproportionate reduction of medial compared with lateral cortex (Pellegrini et al., 1996; Yoshida et al., 1997; present study) may reflect the normal gradient of Emx2 protein, which shows a caudomedial maximum in the embryonic cortex and a rostrolateral minimum (Mallamaci et al., 1998).

The cellular mechanisms that underlie the decreased size of the Emx2 mutant medial cortex, and of the cerebral hemispheres in general, are unclear. A simple explanation might be that Emx2 regulates cortical neuron proliferation and that, in the absence of $E m x 2$, proliferation is reduced. For comparison, smaller cerebral hemispheres are also seen in mice with targeted disruption of the winged helix transcription factor, $B F-1$. In these mice, cerebral hypoplasia results from reduced cell proliferation, together with a precocious neuronal differentiation that depletes the cerebral precursor pool (Xuan et al., 1995). Thus, $B F-1$ mutant embryos have a thinner cerebral VZ than wild-type littermates and a thicker layer of differentiating neurons (Xuan et al., 1995). In contrast, Emx2 mutant embryos have an abnormally thick VZ in the medial embryonic cortex, at least at late stages of corticogenesis, and a thinner, less developed cortical plate.

An alternative explanation, supported by our findings, might be that the Emx2 mutation disrupts the timely maturation of the medial cortex rather than causing a frank decrease in cell proliferation. Such a disruption could reduce the size of the Emx2 mutant cerebral hemispheres and could also have more specific consequences. One example might be the loss of a morphological dentate gyrus in the Emx2 mutant. Although dentate gyrus neurons begin to be generated by VZ cells as early as are CA field neurons, major development of the dentate gyrus occurs in late embryonic and early postnatal life (Angevine, 1965; Caviness, 1973; Stanfield and Cowan, 1979; Altman and Bayer, 1990a,b). Thus, whereas neurogenesis in the mouse CA fields is almost over by E16, neurogenesis in the dentate gyrus increases sharply between E16 and E18 (Caviness, 1973). Because Emx2 mutant mice die at birth, any disruption of the normal schedule of hippocampal development would have a particular impact on the dentate gyrus.

By proliferating and differentiating on an abnormal schedule, cells in the Emx2 mutant cortex might also miss interacting with a variety of cellular and molecular cues that direct specific aspects of their further development. For example, the laminar identity of cortical neurons depends on precisely timed cues within the cortical VZ (McConnell, 1995), and region-specific aspects of cortical development, such as the formation of somatosensory barrel fields, require cues provided by subcortical innervation (O'Leary et al., 1994). If such cues are missed, several specific features of later cortical development could be affected. Whether such effects occur in the Emx2 mutant mouse remains to be determined.

A role for Emx proteins in regulating cortical maturation might also explain the nested expression of Emx genes at the cortical hem. In keeping with a time-limited functional role, the cortical hem region shrinks as the rest of the embryonic cortex expands (Maruyama and D'Agostino, 1967; Zaki and Van der Loos, 1980; Furuta et al., 1997). The selectively low expression in the cortical hem of Emx1 and other genes implicated in tissue growth, such as BF-1 (Xuan et al., 1995) and Lhx2 (Porter et al., 1997) (Fig. 5), could reflect the fate of the cortical hem to shrink with respect to the rest of the cortical mantle. If Emx1 and Emx2 play similar roles, both may be needed to support the normal time course of cortical development, whereas Emx2 alone may be sufficient for the relatively slower growth of the cortical hem.

A final explanation for the reduced size of hippocampal fields in the Emx2 mutant could be that the mutation disrupts the regionalization of medial cortex in a different way from that examined in the present study. Recent evidence indicates that the Emx2 mutation affects neocortical regionalization along the rostrocaudal axis, resulting in an expansion of rostral neocortical areas and a reduction of caudal areas (Bishop et al., 1999). That field boundaries might be shifted in the Emx2 mutant hippocampus, reducing the size of each field, is therefore an intriguing possibility. Because cells are successfully specified to different hippocampal field identities in the Emx2 mutant, this interpretation would dissociate hippocampal patterning into at least two processes; the mechanisms that specify cells to develop particular field identities are not the same as those that specify the overall size of each field. Emx2 is evidently not required for the first process but might be required for the second. However, determining if fields are differentially reduced in the Emx2 mutant according to their position in the hippocampus is difficult given their tiny size. More information may be gained from functional experiments in which Emx proteins are overexpressed in the hippocampus to determine whether these manipulations shift the boundaries of hippocampal fields or alter more general processes of hippocampal growth and maturation.

\section{REFERENCES}

Altman J, Bayer SA (1990a) Migration and distribution of two populations of hippocampal granule cell precursors during the perinatal and postnatal periods. J Comp Neurol 301:365-381.

Altman J, Bayer SA (1990b) Mosaic organization of the hippocampal neuroepithelium and the multiple germinal sources of dentate granule cells. J Comp Neurol 301:325-342.

Altman J, Bayer SA (1990c) Prolonged sojourn of developing pyramidal cells in the intermediate zone of the hippocampus and their settling in the stratum pyramidale. J Comp Neurol 301:343-364.

Angevine JB (1965) Time of neuron origin in the hippocampal region. Exp Neurol Suppl 2:1-70.

Bishop KM, Leingartner A, Richards LJ, O’Leary DDM (1999) Emx2 
imparts area identity to mammalian neocortex. Soc Neurosci Abstr 25:501.

Caviness VS (1973) Time of neuron origin in the hippocampus and dentate gyrus of normal and reeler mutant mice: an autoradiographic analysis. J Comp Neurol 151:113-120.

Caviness VS, Sidman RL (1973) Time of origin of corresponding cell classes in the cerebral cortex of normal and reeler mutant mice: an autoradiographic analysis. J Comp Neurol 148:141-151.

Frantz GD, Bohner AP, Akers RM, McConnell SK (1994) Regulation of the POU domain gene SCIP during cerebral cortical development. J Neurosci 14:472-485.

Furuta Y, Piston DW, Hogan BL (1997) Bone morphogenetic proteins (BMPs) as regulators of dorsal forebrain development. Development 124:2203-2212.

Grove EA, Tole S, Limon J, Yip L, Ragsdale CW (1998) The hem of the embryonic cerebral cortex is defined by the expression of multiple Wnt genes and is compromised in Gli3-deficient mice. Development 125:2315-2325.

Gulisano M, Broccoli V, Pardini C, Boncinelli E (1996) Emx1 and Emx2 show different patterns of expression during proliferation and differentiation of the developing cerebral cortex. Eur J Neurosci 8:1037-1050.

He X, Treacy MN, Simmons DM, Ingraham HA, Swanson LW, Rosenfeld MG (1989) Expression of a large family of POU-domain regulatory genes in mammalian brain development. Nature 340:35-42.

Lee SM, Tole S, Grove EA, McMahon AP (2000) A local Wnt3a signal is required for development of the mammalian hippocampus. Development 127:457-467.

Lumsden A, Krumlauf R (1996) Patterning the vertebrate neuraxis. Science 274:1109-1115.

MacKenzie A, Ferguson MW, Sharpe PT (1991) Hox-7 expression during murine craniofacial development. Development 113:601-611.

Mallamaci A, Iannone R, Briata P, Pintonello L, Mercurio S, Boncinelli E, Corte G (1998) EMX2 protein in the developing mouse brain and olfactory area. Mech Dev 77:165-172.

Maruyama S, D'Agostino AN (1967) Cell necrosis in the central nervous system of normal rat fetuses. An electron microscopic study. Neurology 17:550-558.

McConnell SK (1995) Constructing the cerebral cortex: neurogenesis and fate determination. Neuron 15:761-768.

Motro B, van der Kooy D, Rossant J, Reith A, Bernstein A (1991) Contiguous patterns of c-kit and steel expression: analysis of mutations at the W and Sl loci. Development 113:1207-1221.

Nauta WJH, Feirtag M (1986) Fundamental neuroanatomy. New York: Freeman.

Nicholson-Flynn K, Hitchcock-DeGregori SE, Levitt P (1996) Restricted expression of the actin-regulatory protein, tropomyosin, defines distinct boundaries, evaginating neuroepithelium, and choroid plexus forerunners during early CNS development. J Neurosci 16:6853-6863.

Nowakowski RS, Rakic P (1981) The site of origin and route and rate of migration of neurons to the hippocampal region of the rhesus monkey. J Comp Neurol 196:129-154.
O'Leary DD, Schlaggar BL, Tuttle R (1994) Specification of neocortical areas and thalamocortical connections. Annu Rev Neurosci 17:419-439.

Pellegrini M, Mansouri A, Simeone A, Boncinelli E, Gruss P (1996) Dentate gyrus formation requires Emx2. Development 122:3893-3898.

Porter FD, Drago J, Xu Y, Cheema SS, Wassif C, Huang SP, Lee E, Grinberg A, Massalas JS, Bodine D, Alt F, Westphal H (1997) Lhx2, a LIM homeobox gene, is required for eye, forebrain, and definitive erythrocyte development. Development 124:2935-2944.

Rakic P, Nowakowski RS (1981) The time of origin of neurons in the hippocampal region of the rhesus monkey. J Comp Neurol 196:99-128.

Shigemoto R, Yokota Y, Tsuchida K, Nakanishi S (1990) Cloning and expression of a rat neuromedin $\mathrm{K}$ receptor cDNA. J Biol Chem 265:623-628

Simeone A, Acampora D, Gulisano M, Stornaiuolo A, Boncinelli E (1992a) Nested expression domains of four homeobox genes in developing rostral brain. Nature 358:687-690.

Simeone A, Gulisano M, Acampora D, Stronaiuolo A, Rambaldi M, Boncinelli E (1992b) Two vertebrate homeobox genes related to the Drosophila empty spiracles gene are expressed in the embryonic cerebral cortex. EMBO J 11:2541-2550.

Stanfield BB, Cowan WM (1979) The development of the hippocampus and dentate gyrus in normal and reeler mice. J Comp Neurol $185: 423-459$.

Sturrock RR (1979) A morphological study of the development of the mouse choroid plexus. J Anat 129:777-793.

Theiler K (1989) The house mouse. New York: Springer.

Thomas T, Dziadek M (1993) Capacity to form choroid plexus-like cells in vitro is restricted to specific regions of the mouse neural ectoderm. Development 117:253-262.

Tole S, Grove EA (1998) Early regional patterning of the medial telencephalon. Soc Neurosci Abstr 24:785.

Tole S, Patterson PH (1995) Regionalization of the developing forebrain: a comparison of FORSE-1, Dlx-2 and BF-1. J Neurosci 15:970-980.

Tole S, Christian C, Grove EA (1997) Early specification and autonomous development of cortical fields in the mouse hippocampus. Development 124:4959-4970.

Wisden W, Seeburg PH (1993) A complex mosaic of high-affinity kainate receptors in rat brain. J Neurosci 13:3582-3598.

Xuan S, Baptista CA, Balas G, Tao W, Soares VC, Lai E (1995) Winged helix transcription factor BF-1 is essential for the development of the cerebral hemispheres. Neuron 14:1141-1152.

Yoshida M, Suda Y, Matsuo I, Miyamoto N, Takeda N, Kuratani S, Aizawa S (1997) Emx1 and Emx2 functions in development of dorsal telencephalon. Development 124:101-111.

Zaki W (1981) Ultrastructure of the choroid plexus and its development in the mouse. Z Mikrosk Anat Forsch 95:919-935.

Zaki W, Van der Loos H (1980) [A new concept about the morphogenesis of the prosencephalon in mammals (translated from French)]. Arch Anat Microsc Morphol Exp 69:123-134. 